\title{
Semantic Product Ranking Model (SePRaM) using PNN over the Heuristic Product Data
}

\author{
Gurkiran Kaur \\ Punjabi University Regional Centre for \\ Information Technology and Management, \\ Mohali, Punjab, India
}

\author{
Rekha Bhatia \\ Punjabi University Regional Centre for \\ Information Technology and Management, \\ Mohali, Punjab, India
}

\begin{abstract}
The product ranking models for the e-commerce engines are primarily based upon the various types of product ranking engines based upon collaborative, content-based, hybrid, ordinal, partial and dense ranking models for the realization of the e-commerce product ranking module. The proposed model is based upon the hybridized approach, which is based upon the dual-stage rank preparation. The first stage rank preparation is entirely based upon the content-based ranking model, which evaluates the similarity between the search query arguments and the product ranking data. The product ranking data is prepared by using the various factors associated with the product popularity and accessibility against the search query arguments. The product suggestions are calculated to show the product rankings on the search page to the users. Once the user browsed the specific product, the collaborative classification is used for the higher order product suggestions on the product page. The collaborative approach analyzes the user similarity and produces the product rank lists according to the top listed users in the ranking evaluation. The proposed model evaluation has been analyzed in the form of various time based factors to read the time complexity over the input product data. The proposed model has outperformed the existing models in the terms of the precision and elapsed time.
\end{abstract}

\section{Keywords}

Product ranking model, product recommendation lists, ecommerce product ranking, ranking memory model.

\section{INTRODUCTION}

Ranking system performs comparison between the list of products or items to specify the position of an item. The leading items will be having higher rank and the insignificant item will have given lower rank. With the increase in use of internet people prefer buying products online such as mobile, books, furniture, apparels, etc. From multiple options, ranking system helps the customer to choose the best option.

As e-commerce market is in its preliminary phase, ranking system offers many benefits to the customer:

- It helps the customer to choose the best product among multiple options in lesser time.

- The customer can generate the trust and loyalty relationship with website owners by proving personalized e-commerce ranking system.

- Increases competition among different companies by comparing their products which helps to improve the quality and services of product.

To rank the product, firstly the products are divided in subcategories and each category is ranked identically. For example, laptops are categories according to their companies like apple, Samsung, hp, dell, etc. the companies are divided into the sub-categories like model names and at each step ranking is performed.

E-commerce means electronic commerce. It provides the services and products to the customer by using the network such as internet. Through e-commerce, the market can be accessed globally. E-commerce is used for bill payment, email, ebooks, online shopping, etc. these services are provided by various companies such as snapdeal, quikr, flipkart, amazon, paytm and olacabs. E-commerce improves level of customer services and it reduces the transaction cost. The ecommerce can be classified into different sub-categories such as B2C or Business-to-Consumer, B2B or Business-toBusiness, B2G or Business-to-Government, $\mathrm{C} 2 \mathrm{C}$ or Consumer-to-Consumer, B2E or Business-to-Employee, C2B or Consumer-to-Business. E-commerce ranking based approaches are:

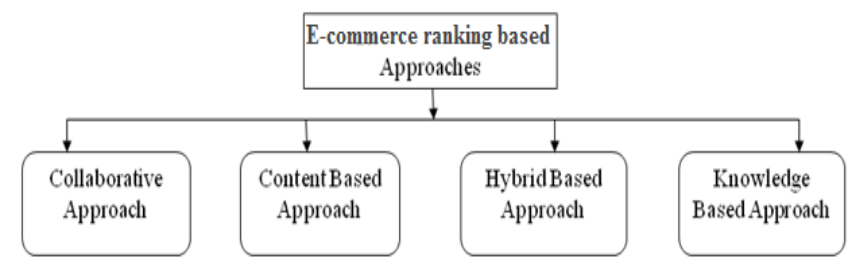

- There are different types of approaches of recommender systems that vary in terms of the knowledge used, the recommendation algorithm followed, how the Ecommerce ranking are generated, assembled and presented to the user

- There are four basic techniques as mentioned in the figure

Collaborative filtering: is a popular approach

- Key assumption behind this type of E-commerce ranking system is that it might happen that people related to each other might have same likings and interest

- Other users with same likings and interest are selected and by using their data and ratings the E-commerce ranking system to the active user is made

\section{Content based recommendation:}

- Evolution of the content based E-commerce ranking system started from the concepts of information retrieval and information filtering research

- Predicts and indexes the item to the user by evaluating the content of the items and profile provided by the user

Hybrid approach: combination of two or more techniques 
- Increases the efficiency of recommender systems

- Incorporating the results of collaborative and contentbased filtering creates the potential for a more accurate recommendation

- Hybrid approach could also be used to address collaborative filtering problem of sparse data known as cold start

\section{Knowledge based recommendation:}

- In such knowledge-based approaches, the recommender system typically makes use of additional, often manually provided, information about both the current user and the available items

- Constraint based recommenders are one example of such systems

Semantic web is used to gather data in the computer system without the instruction of human and present it to the user in a human readable form in the form of web pages.Firstly it creates the web data stores on web and develops the common framework that allows to distribute the data among companies, applications or communities. The semantic web is integrated with the ranking system and after that dynamic amalgamation is performed. In dynamic amalgamation, if a new product is added in a ranked list then, its comparison with other products in the list is performed and the rank is generated automatically. After that the whole list is updated.. In ranking system, multiple factors are considered to rank the product. The following factors will be used for evaluation the ranking system are:

Alexa rank: It is used to calculate the website rank. Alexa rank is generated by Alexa website and it can track the page view per user, user location, etc. It is the easiest way to known status of the multiple websites.

Google Page Rank: Google Page Rank (PR) is another website ranking model by the Google Inc. It is measured on the scale of 0 to 10 and includes the quality backlinks to the page or website.

Alexa Trust Rank: The material provided by the Alexa Rank is used to calculate the Alexa trust. In this the trust factor evaluates the Daily time on site, daily Perviews per visitor, bounce rate, etc. The trust factor gives the reliability of the websites for its users.

Google Trust Factor: Google's trust factor integrates several factors to generate a value to know how much trustful a particular site is. The articles of the more trustful site will be ranked higher on specific Google searches. Harmful and/or low quality content can make the site less trustful.

The section 2 describes the literature study over the existing ecommerce methods, machine learning and ranking systems. The section 3 describes the experimental design of the proposed model that has been designed for the product ranking evaluation over the e-commerce products. The section 4 includes result analyses that validates the problems and compare the existing model with the proposed model. Section 5 describes the conclusion by concluding the factors imposed and studied in all of the previous sections.

\section{LITERATURE REVIEW}

Neha verma et.al.[1] have designed an algorithm known as "SNEC page ranking algorithm" based on semantic and neural based ranking algorithm. The author has combined the ecommerce with web mining. The algorithm is helpful for the customers to choose the best option among the multiple options and help the companies to know their weakness and present the new improvised product to the customer. The SNEC algorithm is used as a website ranking tool and website has been ranked with the help of various suitable features. The results are accurate but can be improved by incorporating more features into it.

Tejeda-Lorente, Álvaro et.al. [2] have designed a recommender system that refine and analyze the huge amount of data available on web which helps the user to achieve the suitable information according to their search. In this paper, the author has proposed the recommender system that focuses on a quality instead of features of an item and user profile . The recommender system is designed with the help of fuzzy linguistic approach and tested in a digital library of a university.

Hepp, Martin et.al. [3] have worked for Researchers and Practitioners on the Web of Data for E-Commerce particularly for Schema.org and GoodRelations. They have develop the different patterns for the requirements and ownerships which include variety of products like books, cosmetics, electronic devices, furniture, apparels, etc and have created a full tool chain for generating and engrossing the respective data for providing the guidance on the conceptual structure of the schema.org. Also the authors have explained the long-term vision of Linked Open Commerce, and discussed advanced topics, like identity and authentication, access control, (e.g. with WebID); data management issues from the publisher and consumer perspective and micropayment services. The authors have also covered research opportunities resulting from the growing adoption and the specific amount of data in Microdata, RDFa, and JSON-LD syntaxes. But this application not applicable for e-commerce product ranking system as it is restricted to micro-data.

Na chan, Viktor k. prasanna et.al. [4] has introduced the rankbox which is a ranking system on a semanticweb for mining the complex relationship. The aim of the paper is to provide personalized results to end user based on user preferences that are achieved from their feedback generated from search results and produced more precise results. The author also tries to conceal the technical details from end user. The quality of ranking system is improved by generating more feedbacks. The rankbox system is used with the web browsers by the different devices. The final result indicates the overall benefits and potency of the system.

Shiguag Ju, Zheng Wang et.al. [5] has improved page rank algorithm using timestamp and link. In this paper the author had tried to resolve the problem appears in the traditional ranking algorithms where the old pages are always provided with the higher rank by static ranking algorithm in accordance with dynamic web. The author presented the temporal-linkanalysis algorithm in which HTTP response is used to return the utmost modification time as a timestamp of the concerned links and nodes. The weight of the page is calculated by combining the in-link and out-links. The new algorithm i.e WTPR algorithm is compared with the other algorithms and the results shows that the new algorithm is more effective and the new and old pages are ranked according to their effectiveness of the pages and produce accurate ranking.

Upal Senanyak, Peter Scot et.al. [6] have worked on page rank algorithm to analyze its performance using the different network structures such as random networks with a custom search engine and scale free networks. The performance of the algorithm is evaluated under various topological conditions. It 
has been observed that the performance of page rank algorithm was declined due to disturbance in random network as they are sensitive. The real life networks can be incomplete which can cause disturbance for the complete network and increase the risk in ranking the nodes. Whereas the free scale network produce the constant results and show the less disturbance over multiple networks. So, to maintain the effectiveness of page rank algorithm it should not lose its free scale nature.

The section 3 describes the experimental design for the product ranking analysis over the e-commerce products.

\section{EXPERIMENTAL DESIGN}

The proposed model has been designed for the product ranking evaluation over the e-commerce products. The ecommerce portals require the several types of the product lists to be shown on the different page models. The primary page models include the main page, product page, etc where the product lists are required to be shown particularly. The content based engine is based upon the input query whereas the collaborative engine evaluates the similarity between the querying user and other users using the feed forward probabilistic neural network (PNN) classification. The probabilistic neural network classifies the testing data against the training data of the user profiles. The similarity evaluation is based upon the entity weight calculation and automatic inclusion of the product entity evaluation for the product list ranking. The detailed ranking algorithm has been shown in the following section:

\section{Algorithm 1: HYBRID PRODUT RANKING MODEL}

Input: \{Product Data, Popularity Factors (Local and Global), user data $\}$

Output: Product Rank List

1. Call the e-commerce API for product data acquisition

2. Extract each individual entity and prepare the initial product data matrix

3. Calculate the size of the product data matrix

4. Start the iterative function over the product data matrix

a. Obtain each rows data on each iteration from the product data matrix

b. Extract the local and global factors from the input data matrix

c. Compute the input factors to prepare the generalized average value (GAV)

d. Add the GAV to the product ranking list (PRAL)

e. Add the product id to the PRAL

f. If the iteration count equals number of rows

i. Return the iteration

ii. Return the PRAL matrix

g. Switch to the step 4(a) otherwise

5. Input the browsing history of all users

6. Obtain the current user history data

7. If history data list is empty

8. Else

a. Return PRAL as final product rank matrix

a. Read the user browsing history and arrange the data according to timeline b. Create the four-way preliminary user priority matrix (FPUPM) populated with product and manufacturer rating, total access and purchases

c. Set up the neural network arguments

d. Input the number of network layers

e. Create the neural network

f. Initialize the neural network activation function

g. Acquire the user browsing data

h. Rearrange the user history data according to the four-way matrix for the training input to the neural network

i. Run the neural network over the training and testing data

j. Return the neural network classification decision

k. Sort the similar user list in descending order

1. Obtain the top rows

m. Apply column wise averaging factors over the result matrix

n. Create the user similarity averaging vector (SAV)

o. Return SAV

9. Collaborate the PRAL and SAV matrices

10. Apply the resorting procedure over the final collaborative matrix (FCM)

11. Return the FCM

The section 4 describes the result analysis obtained by implementing the proposed model that is mentioned in previous section and by comparing it with the existing models.

\section{RESULT ANALYSIS}

The time based comparison between the existing and proposed model has been done on the basis of the total time to compute the results. Here in this time analysis graph the existing model is shown with blue color and proposed model with red color. The proposed model has performed the task in merely 0.1 seconds against the existing model time of 0.9 seconds, which clearly signifies the quickness of the proposed model to compute the results quicker.

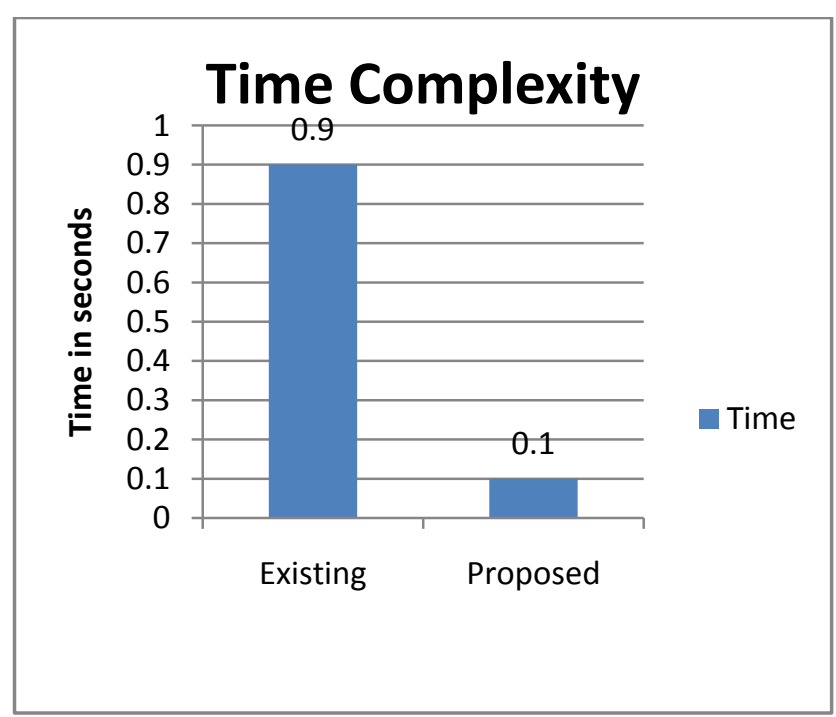

Figure: 4.1: Time based analysis of the proposed and existing model for the ranking model 
The time based analytical comparison between the existing and proposed models clearly indicates the robustness of the proposed model in computing the result faster and quicker. The time complexity based evaluation is usually helpful to understand the time expensive bottlenecks due to the heavy data processing events. The proposed model has been deeply analyzed for the time complexity parameters. The following table 4.1 shows the time complexity results obtained from both of the models.

Table: 4.1: The content and collaborative ranking based comparative analysis based upon time complexity

\begin{tabular}{|l|l|l|l|l|}
\hline $\begin{array}{l}\text { Iteratio } \\
\text { n Count }\end{array}$ & $\begin{array}{l}\text { Existing } \\
\text { Content- } \\
\text { based } \\
\text { Ranking }\end{array}$ & $\begin{array}{l}\text { Existing } \\
\text { Collaborati } \\
\text { ve Ranking }\end{array}$ & $\begin{array}{l}\text { Proposed } \\
\text { Content- } \\
\text { based } \\
\text { Ranking }\end{array}$ & $\begin{array}{l}\text { Proposed } \\
\text { Collabora } \\
\text { tive } \\
\text { Ranking }\end{array}$ \\
\hline $\mathbf{1}$ & 0.3096 & 0.89978 & 0.0232 & 0.09508 \\
\hline $\mathbf{2}$ & 0.3124 & 0.8564 & 0.0233 & 0.08302 \\
\hline $\mathbf{3}$ & 0.3087 & 0.8765 & 0.0213 & 0.09212 \\
\hline $\mathbf{4}$ & 0.3698 & 0.9644 & 0.0232 & 0.09014 \\
\hline $\mathbf{5}$ & 0.3310 & 0.8761 & 0.0241 & 0.10200 \\
\hline $\mathbf{6}$ & 0.3091 & 0.8315 & 0.0201 & 0.08120 \\
\hline $\mathbf{7}$ & 0.3265 & 0.9216 & 0.0234 & 0.09200 \\
\hline $\mathbf{8}$ & 0.3425 & 0.8917 & 0.0233 & 0.09519 \\
\hline
\end{tabular}

The proposed model results have been obtained in the form of the time complexity obtained from both of the models. The proposed model produces the content-based and collaborative ranking faster than the existing model, which is clearly shown from the table 4.1. The time complexity results have been obtained in the seconds, and there is the difference of almost 10 times less time than the existing model's calculated time.

Table 4.2: The precision based result evaluation

\begin{tabular}{cccc}
\hline Iteration & $\begin{array}{c}\text { Website Priority } \\
\text { Tool }\end{array}$ & Google & Proposed \\
\hline 1 & 1.5 & 1.9 & 1.75 \\
2 & 2.65 & 2.3 & 2.7 \\
3 & 2.48 & 2.1 & 2.58 \\
\hline
\end{tabular}

The table 4.2 shows the results obtained from the existing tools based upon the product ranking, which includes the existing web priority tool, Google and the proposed model. The proposed model has been marginally improved than the existing models on the second and third iteration, which signifies the stronger results obtained from the proposed model in accordance with the existing model. The proposed model has been found more robust and accurate in producing the results as it as shown the higher precision in the obtained results than the existing models. The marginal improvement in the ranking model may show the large differences in the performance when incorporated on the large scale applications.

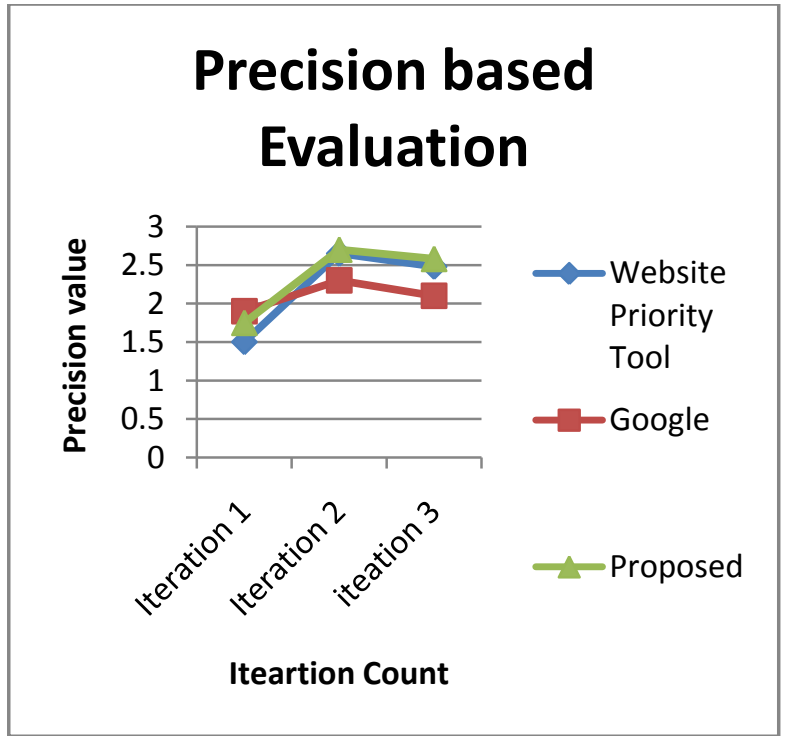

Figure 4.2: The precision based evaluation of the proposed model

The figure 4.2 graphically shows the results obtained from the table 4.2. The graphical plot of all of the models under the comparative analysis has been evaluated thoroughly on the basis of the precision of the calculated results. The proposed model lies between the Google and Web priority tool results in initial stage, whereas it improves the further iterations. The marginal improvement has been recorded which justifies the improved performance of the proposed model than the existing models.

The section 5 describes the conclusion obtained after the complete study of the ranking system and result that are obtained by proposed model.

\section{CONCLUSION}

The proposed model has been designed for the e-commerce portals for the inclusion of the product ranking on the basis of the factors used to signify the popularity or accessibility. The proposed model includes the dual-stage ranking model, which combines the collaborative ranking along with the contentbased ranking model. The content-based ranking is computed over the given data against the input search query, whereas the collaborative approach utilizes the feed forward probabilistic neural network (FFPNN). The proposed model performance has been evaluated under the various experiments conducted to analyze the overall performance in the terms of accuracy and time complexity. The proposed model has been recorded for the recall rate for the measure of the accuracy. Also, it has been studied for the hybrid time response along with the model ranking time divided for the content-based and collaborative ranking models over the proposed and existing methods. The results clearly show the improvement in the proposed model results obtained from the several experiments, when compared to the prominent existing models for the ecommerce ranking.

\section{REFERENCES}

[1] Verma, Neha, Dheeraj Malhotra, Monica Malhotra, and Jatinder Singh. "Online Libraries Website Recommendation Using Semantic Web Mining and Neural Computing." Procedia Computer Science 45 (2015): pp. 42-51, ELSEVIER. 
[2] Hepp, Martin. "The Web of Data for Online Libraries: Schema. org and GoodRelations for Researchers and Practitioners." In Engineering the Web in the Big Data Era, pp. 723-727.Springer International Publishing, 2015.

[3] Tejeda-Lorente, Álvaro, Carlos Porcel, Eduardo Peis, Rosa Sanz, and Enrique Herrera-Viedma. "A quality based recommender system to disseminate information in a university digital library." Information Sciences 261 (2014): 52-69.

[4] Chen, Na, and Viktor K. Prasanna. "Rankbox: An adaptive ranking system for mining complex semantic relationships using user feedback." InInformation Reuse and Integration (IRI), 2012 IEEE 13th International Conference on, pp. 77-84. IEEE, 2012.

[5] Ju, Shiguang, Zheng Wang, and Xia Lv. "Improvement of page ranking algorithm based on timestamp and link." In Information Processing (ISIP), 2008 International Symposiums on, pp. 36-40. IEEE, 2008.

[6] Senanayake, Upul, Peter Szot, Mahendra Piraveenan, and Dharshana Kasthurirathna. "The performance of page rank algorithm under degree preserving perturbations." In Foundations of Computational Intelligence (FOCI), 2014 IEEE Symposium on, pp. 24-29. IEEE, 2014.

[7] Sessoms, Matthew, and KemaforAnyanwu. "Enabling a Package Query Paradigm on the Semantic Web: Model and Algorithms."In Transactions on Large-Scale Dataand Knowledge-Centered Systems XIII, pp. 1-32.Springer Berlin Heidelberg, 2014.

[8] Malhotra, Dhairya. "Intelligent web mining to ameliorate Web Page Rank using Back-Propagation neural network." In Confluence The Next Generation
Information Technology Summit (Confluence), 2014 5th International Conference-, pp. 77-81. IEEE, 2014.

[9] Furukawa, Takao, Kaoru Mori, KazumaArino, Kazuhiro Hayashi, and Nobuyuki Shirakawa. "Identifying the evolutionary process of emerging technologies: A chronological network analysis of World Wide Web conference sessions."Technological Forecasting and Social Change 91 (2015): 280-294.

[10] Scioscia, Floriano, Michele Ruta, Giuseppe Loseto, Filippo Gramegna, Saverioleva, Agnese Pinto, and Eugenio Di Sciascio. "A Mobile Matchmaker for the Ubiquitous Semantic Web." International Journal on Semantic Web and Information Systems (IJSWIS) 10, no. 4 (2014): 77-100.

[11] Mital, Monika, AshisPani, and Ram Ramesh. "Determinants of choice of semantic web based Software as a Service: An integrative framework in the context of e-procurement and ERP." Computers in Industry 65, no. 5 (2014): 821-827.

[12] D.T. Green and J. M. Pearson, "The examination of two web site usability instruments for use in B2C Online Libraries organizations," Journal of ComputerInformation Systems, Vol. 49, No. 4, 2009, pp. 19-32

[13] T. Wang and Y. Lin, "Accurately predicting the success of B2B ecommerce in small and medium enterprises," Expert Systems withApplications, Vol. 36, No. 2, published by Elsevier, 2009, pp. 2750-2758.

[14] M. Lazarica and I. Lungu, Aspecteprivindproiectareasistemelor de comert electronic, ASE Publishing House, 2007,pp. 147. 